should be ordered full doses of quinine and colchicum. Mr. Jacobson ${ }^{4}$ in a lecture on the same subject also insists upon the necessity for a prompt suppression of the urethral discharge. To produce this result he prefers for the first few days the injection of boiled water, afterwards ordinary Watson Cheyne's bougies -one to be introduced and retained for half an hour twice daily. In the case of gonorrhoal arthritis he advocates fixation with gentle pressure to the affected joint and the improvement of the patient's general vigour and vitality. The former result is best secured by plaster of Paris applied when necessary over pads of cotton-wool and followed in due course by a Martin's rubber bandage. Very rarely indeed does gonorrhœal arthritis call for anything in the shape of heroic surgical measures, though occasionally obstinate persistence of synovial fluid may need paracentesis or incision. Generous feeding, together with such tonics as cod-liver oil, strychnine, and the hypophosphites, is essential to meet the bodily and mental depression which almost invariably attend this disease.

1 Poly clinic, July 1904. 2 Medical Review, vol. ii., p. 160. 3 Lancet, July 9th, 1904. 4 Polyclinic, April 1902.

\section{RHEUMATISM AS A CAUSE OF EPISTAXIS IN CHILDREN.}

THE study of rheumatic manifestations, as these occur in the early years of life, has effected something like a revolution in the clinical presentation of the disease as compared with the teaching generally prevalent, say, a generation ago. Instead of being regarded as mainly an affection of the joints, with a greater or less liability to cardiac valve disease-the picture presented by the rheumatic adult -acute rheumatism is seen, when studied in the child, to have a much wider and more varied range of phenomena. Erythemas, tonsillitis, chorea, purpura, subcutaneous nodules, perhaps also pleurisy and other localised inflammatory processes, are now commonly recognised as members of the rheumatic series, and are more frequent as exhibitions of the disease in early life than the acute polyarthritis which is the limited display of the disease to which the adult is mainly liable. The tendency is to add to the number of incidents having a rheumatic basis. Recently ${ }^{1}$ attention was directed in these columns to abdominal pain in children as an exexpression of rheumatism. It is also to be noted that the same claim has been advanced for epistaxis. Dr. Sydney Phillips ${ }^{2}$ was one of the earliest observers to draw attention to this point. $\mathrm{He}$ published a series of cases, in some of which the epistaxis came on with an attack of acute rheumatism, whilst in others the bleeding occurred in the intervals between articular inflammations, chorea, or other recognised rheumatic incidents. Dr. Phillips suggested that in every case of epistaxis in early life care should be taken to examine the joints, as undoubtedly in children synovial effusion and articular rheumatism may occur without pain. It has been charged against the salicylates that they sometimes cause epistaxis; but, in view of the above records and of the fact that none of the patients had taken salicylates prior to the epistaxis, it becomes a question whether the result attributed to the remedy ought not to be debited to the disease. Dr. Graham Lang will ${ }^{3}$ has also described attacks of epistaxis in rheumatic children. In one of his cases the bleeding occurred at each of three separate attacks of acute rheumatism. There cannot be much difficulty in accepting the clinical position suggested by these records. If rheumatism, as few deny, is capable of producing subcutaneous hæmorrhages as in purpura rheumatica it is not difficult to conceive that similarly it may produce epistaxis. And it may be that the epistaxis which is occasionally noted in cases of mitral obstruction, and is then attributed to the valvular lesion, has a more direct relationship to the rheumatic process. In a recent article Dr. Herbert Tilley ${ }^{4}$ accepts the position that epistaxis may be in children an expression of rheumatism, and claims that treatment by salicylates is successful in preventing recurrence.

1 The Hospital, June 18, 1904. 2 The Lancet, Feb. 22, 1902. 3 Scottish Med. \& Surg. Jour., Nov. 1903. 4 Clin. Jour., June 1, 1904.

\section{PRIMARY MUSCULAR TUBERCULOSIS. ${ }^{1}$}

Primary tuberculosis of muscles is a clinical term rather than an exact pathological definition. The tuberculous foci, it is true, are embedded in the muscle and have the general clinical characters of muscle tumours, but their point of origin is the connective tissue sheath, not the muscle. Again, though described as primary to distinguish it from a secondary tuberculous myositis due to extension by contiguity of tissue, the condition probably always follows an initial lesion, which, however, may be so remote and obscure that the tuberculosis of the muscle constitutes for all practical purposes a distinct morbid entity. Three varieties of the disease are described, namely, the gumma, the cold abscess, and the tuberculous infiltration, the last-named being the most infrequent of the three. The cold abscess is the most common form, and it has to be distinguished from hydatid cyst, sarcoma, lipoma, and syphilitic gumma; also it must be remembered that a chronic, indolent, intra-muscular tumour may be a non-tuberculous abscess due to the suppuration of an old hæmatoma, or an abscess containing a culture of the Eberth bacillus and following enteric fever. In other cases there are numerous tumours widely spread over the body and often appearing at or about the same date. The diagnosis in these instances is helped by the different consistence of the several tumours, some being soft and fluctuant, while others are firm and resistant. If necessary, an exploratory puncture will help to distinguish the condition from sarcomatosis, multiple hydatid cysts, and from actinomycosis. In spite of the evidence of widespread infection in these cases, the patients often show little depreciation of the general health, unless at the same time they are the victims of pulmonary or other serious form of tuberculosis. The tumours ought always to be extirpated, whether multiple or single, and the operation should be a thorough one, not mere scraping or curettage, but complete ablation, removing, where necessary, considerable masses of muscle. If left without treatment, these tuberculous nodules tend to perforate the aponeuroses and spread into the subcutaneous tissues, or to run along the tendons and invade the synovial sacs. The operation not only avoids these risks, but is usually followed by im- 